\title{
Growth and Characterization of Non-Linear Optical Single Crystal: L-cysteine Hydrochloride Monohydrate
}

\author{
V. AzeezaA ${ }^{a, b}$, A.J. Arul Pragasam ${ }^{a}$, T.G. Sunitha ${ }^{c}$, P. Koteeswari ${ }^{d}$ And S. Suresh ${ }^{e, *}$ \\ ${ }^{a}$ Department of Physics, Sathyabama University, Tamilnadu-600 119, India \\ ${ }^{b}$ Department of Physics, SRR Engineering College, Tamilnadu-603 103, India \\ ${ }^{c}$ Department of Chemistry, Pachaiyappa's College, Tamilnadu-600 030, India \\ ${ }^{d}$ Department of Physics, Justice Basheer Ahmed Sayeed College for Women, Chennai 600018, India \\ ${ }^{e}$ Department of Physics, AMET University, Chennai 603 112, India
}

(Received January 28, 2015; in final form September 25, 2015)

\begin{abstract}
Nonlinear optical single crystals of L-cysteine hydrochloride monohydrate (LCB) were grown by slow evaporation technique. Single crystal X-ray diffraction analysis revealed the crystal system and helped to determine lattice parameter values. Powder X-ray diffraction analyses were carried out and the diffraction patterns were indexed. The optical properties of the crystals were determined using UV-visible spectroscopy. Optical constants such as refractive index, extinction coefficient and electric susceptibility were determined from UV-visible spectroscopy. The Fourier transform infrared studies confirmed the various functional groups present in the grown crystal. The mechanical behaviour of the grown crystals was studied using Vicker's microhardness tester. The thermal analysis confirmed that the crystal was stable up to $108.7^{\circ} \mathrm{C}$. The dielectric constant and the dielectric loss measurements were carried out for different temperatures and frequencies. Second harmonic generation of LCB crystal was investigated by the Kurtz powder technique.
\end{abstract}

DOI: 10.12693 /APhysPolA.128.423

PACS: 81.10.Dn, 78.20.Ci, 67.55.Hc

\section{Introduction}

In recent years there has been considerable interest in synthesizing non-linear optical materials with excellent second order optical non-linearities owing to their potential application in electro-optic and opto-electronic devices $[1,2]$. Non-linear optics (NLO) is at the forefront of current research because of its importance in providing the key functions of frequency shifting, optical modulation, optical switching, optical logic and optical memory for the emerging technologies in the areas such as telecommunications, signal processing and optical interconnections $[3,4]$.

The rapid development of optical communication systems has led to a demand for non-linear optical materials with high optical quality. Owing to the technological importance of these non-linear crystals, the need for good quality crystals has grown dramatically in the last few decades. The wide range of applicability of bulk single crystals is evident in the fields of semiconductors, infrared detectors, non-linear optics, piezoelectric oscillators, photonics and optoelectronic industries. The key factors for materials selection depend not only on the laser condition but also on the physical properties of the crystal such as transparency, damage threshold, conversion efficiency, phase matching, temperature stability, and size of the crystal [5]. Slow evaporation methods, slow cooling method etc., are known for single crystal growth tech-

${ }^{*}$ corresponding author; e-mail: sureshsagadevan@gmail.com niques from solution [6-8]. Solution growth technology plays a principal role for the non-linear optical susceptibilities. The transparency and other mechanical properties of the grown crystals are affected in other growth processes due to thermal stress. It is reported that solution growth overcomes these difficulties due to its simplicity and convenience [9].

The borate crystals generally possess chemical stability, high damage threshold and high optical quality [10]. The experiment conducted by Becker on NLO materials proved that borate compound materials are superior to other commonly used materials for UV applications [1113]. Moreover, amino acids are dipolar in nature and the molecules possess an electron donor group and an electron acceptor group. This leads to large second order optical non-linearity arising out of intramolecular charge transfer between the donor and the acceptor. Owing to dipolar nature, amino acids are considered to be high potential for NLO applications [14].

Some of the L-cysteine complexes are L-cysteine tartarate monohydrate [15], L-cysteine hydrochloride monohydrate $[16,17]$ and L-cysteine hydrochloride [18]. In the present investigation, attempts were made to grow single crystals of L-cysteine hydrochloride monohydrate (LCB) by slow solvent evaporation technique and characterization on single crystal X-ray diffraction (XRD), UVvisible absorption, the Fourier transform infrared (FTIR) spectroscopy, microhardness, thermogravimetricdifferential thermal analysis (TG-DTA), differential scanning calorimetry (DSC), second harmonic generation and dielectric studies were done as well. 


\section{Growth of LCB single crystal}

Single crystals of L-cysteine hydrochloride monohydrate were formed by dissolving commercially available L-cysteine hydrochloride monohydrate in double distilled water using slow evaporation technique. The solution was stirred continuously for $5 \mathrm{~h}$ using a magnetic stirrer to obtain a homogeneous mixture over the entire volume of solution. The prepared solution was filtered and kept undisturbed at room temperature. Tiny seed crystals with good transparency were obtained due to the spontaneous nucleation. Among them, defect free seed crystal was suspended in the mother solution, which was allowed to evaporate at room temperature. Large size single crystals were obtained due to the collection of monomers at the seed crystal sites from the mother solution, after the nucleation and growth processes were completed. LCB crystal of dimension about $20 \times 11 \times 8 \mathrm{~mm}^{3}$ was harvested in a growth period of 35 days by slow evaporation of the solvent. The photograph of the grown LCB crystal is shown in Fig. 1.

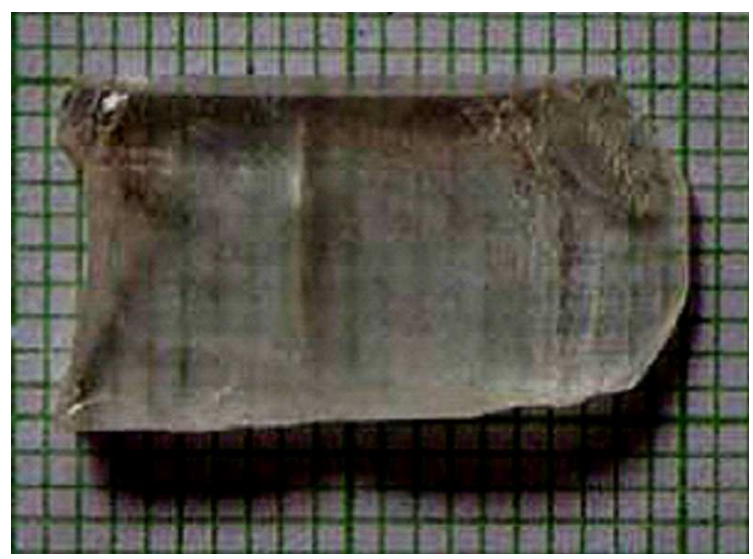

Fig. 1. Photograph of LCB.

\section{Results and discussions}

\subsection{Single crystal X-ray diffraction analysis}

Single crystal X-ray diffraction analysis for the grown crystals LCB was carried out to identify the cell parameters using an MESSRS ENRAF NONIUS CAD 4, The Netherlands, automatic X-ray diffractometer. The lattice parameters were calculated by using least-squares refinement and were found to be: $a=$ $19.43 \AA, b=7.21 \AA, c=5.54 \AA$ and the crystal belonged to orthorhombic system and space group $P 2_{1} 2_{1} 2_{1}$ which agreed well with the reported values [19].

\subsection{Powder X-ray diffraction studies}

The powder X-ray diffraction study was performed on the grown crystal to determine its lattice parameters. The crystal was scanned over the range $20^{\circ}-70^{\circ}$ at a rate of $1^{\circ}$ per minute. The lattice parameters and the cell volume obtained from powder XRD were: $a=19.43 \AA$, $b=7.21 \AA, c=5.54 \AA$ and the crystal belonged to orthorhombic crystal system and space group $P 2_{1} 2_{1} 2_{1}$. The peaks obtained from powder XRD were indexed using UNIT CELL program. The indexed powder pattern is shown in Fig. 2. The results agreed well with single crystal XRD results.

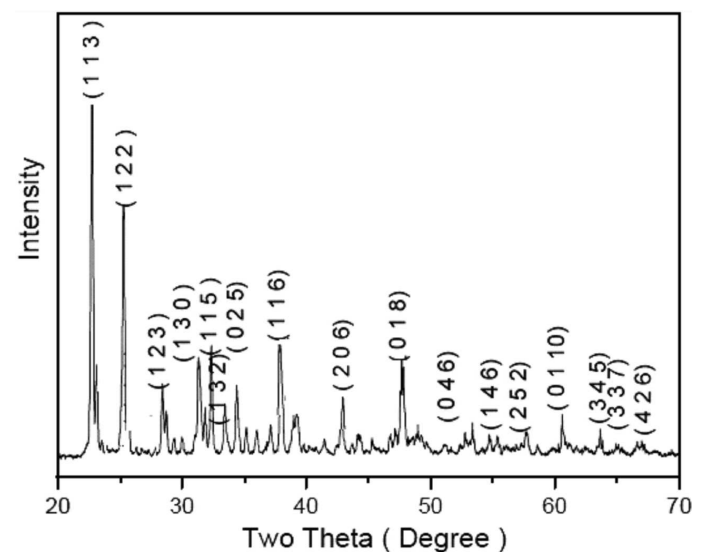

Fig. 2. Powder X-ray diffraction pattern of LCB single crystal.

\subsection{UV-visible spectroscopy}

The optical transmission spectrum of LCB single crystal was recorded in the wavelength region 200-1400 nm and it is shown in Fig. 3a. For optical fabrications, the crystal should be highly transparent in the considered region of wavelength $[20,21]$. The favorable transmittance of the crystal in the entire visible region suggests its suitability for second harmonic generation. The UV absorption edge for the grown crystal was observed to be around $255 \mathrm{~nm}$. The dependence of optical absorption coefficient on photon energy helps to study the band structure and type of transition of electrons. The optical absorption coefficient $(\alpha)$ was calculated from transmittance using the following relation [22]:

$$
\alpha=\frac{1}{d} \ln \left(\frac{1}{T}\right),
$$

where $T$ is the transmittance and $d$ is the thickness of the crystal. The crystal under study had an absorption coefficient $(\alpha)$ obeying the following relation for high photon energies $(h \nu)$ :

$$
\alpha=\frac{A\left(h \nu-E_{\mathrm{g}}\right)^{1 / 2}}{h v},
$$

where $E_{\mathrm{g}}$ is the optical band gap of the crystal and $A$ is a constant. A plot of variation of $(\alpha h \nu)^{2}$ versus $h \nu$ is shown in Fig. 3b. $E_{\mathrm{g}}$ was evaluated using the extrapolation of the linear part [23]. Using the Tauc plot, the energy gap $\left(E_{\mathrm{g}}\right)$ was calculated to be $4.80 \mathrm{eV}$ and the large band gap clearly indicated the wide transparency of the crystal [24]. This high band gap value indicated that the grown crystal possessed dielectric behaviour to induce polarization when powerful radiation was incident on the material. 

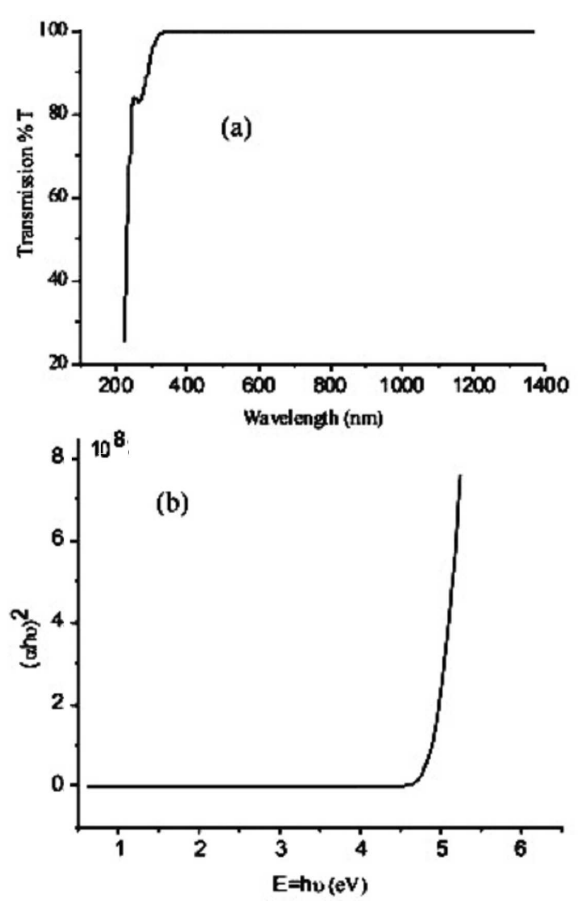

Fig. 3. (a) UV-vis spectrum, (b) plot of $(\alpha h \nu)^{2}$ vs. photon energy $h \nu$ of LCB.

\subsubsection{Determination of optical constants}

Two of the most important optical properties, viz., the refractive index and the extinction coefficient are generally called optical constants. The amount of light that transmits through crystal depends on the amount of the reflection and the absorption that take place along the light path. The optical constants such as the refractive index $(n)$, the real part of dielectric constant $\left(\varepsilon_{\mathrm{r}}\right)$ and the imaginary part of dielectric constant $\left(\varepsilon_{\mathrm{i}}\right)$ were determined. The extinction coefficient $(K)$ could be obtained from the following equation:

$$
K=\frac{\lambda \alpha}{4 \pi} \text {. }
$$

The extinction coefficient $(K)$ was found to be $3.12 \times 10^{-6}$ at $\lambda=1400 \mathrm{~nm}$. The transmittance $(T)$ is given by

$$
T=\frac{(1-R)^{2} \exp (-\alpha t)}{1-R^{2} \exp (-2 \alpha t)} .
$$

Reflectance $(\mathrm{R})$ in terms of absorption coefficient was obtained from the above equation [25]. Hence,

$$
R=\frac{1 \pm \sqrt{1-\exp (-\alpha t+\exp (\alpha t)}}{1+\exp (-\alpha t)} .
$$

Refractive index $(\mathrm{n})$ could be determined from reflectance data using the following equation,

$$
n=-\frac{(R+1) \pm \sqrt{3 R^{2}+10 R-3}}{2(R-1)}
$$

The refractive index $(n)$ was found to be 1.25 at $\lambda=$ $1400 \mathrm{~nm}$. From the optical constants, electric susceptibility $\left(\chi_{\mathrm{c}}\right)$ could be calculated using the following relation [26]:

$$
\varepsilon_{\mathrm{r}}=\varepsilon_{0}+4 \pi \chi_{\mathrm{c}}=n^{2}-k^{2} .
$$

Hence,

$$
\chi_{\mathrm{c}}=\frac{n^{2}-k^{2}-\varepsilon_{0}}{4 \pi},
$$

where $\varepsilon_{0}$ is the permittivity of free space. The value of electric susceptibility $\left(\chi_{\mathrm{c}}\right)$ was 0.214 at $\lambda=1400 \mathrm{~nm}$. The real part dielectric constant $\left(\varepsilon_{\mathrm{r}}\right)$ and the imaginary part dielectric constant $\left(\varepsilon_{\mathrm{i}}\right)$ could be calculated from the following relations:

$$
\begin{aligned}
\varepsilon_{\mathrm{r}} & =n^{2}-k^{2}, \\
\varepsilon_{i} & =2 n k .
\end{aligned}
$$

The values of real dielectric $\left(\varepsilon_{\mathrm{r}}\right)$ and the imaginary constant $\left(\varepsilon_{\mathrm{i}}\right)$ at $\lambda=1400 \mathrm{~nm}$ were estimated at 1.345 and $4.785 \times 10^{-5}$, respectively. The moderate values of the refractive index and the optical band gap suggest that the material has the required transmission range for NLO application. The lower value of dielectric constant and the positive value of the material enable the occurrence of induced polarization due to intense incident light radiation. The optical conductivity is one of the powerful tools for studying the electronic states in materials. The frequency dependence of the dielectric indicates that a material's polarization does not respond instantaneously to an applied field. For this reason, dielectric constant is often treated as a complex function of the frequency of the applied field. A perfect dielectric is a material that has no conductivity. However, the grown crystals associated with low dielectric loss inhibit the propagation of electromagnetic energy which aided conductivity. The optical conductivity $(\sigma)$ of the crystal was calculated using the following relations [27-30]:

$$
\sigma=\frac{\omega}{4 \pi} \operatorname{Im}(\varepsilon),
$$

where the value of $\operatorname{Im}(\varepsilon)$ is given by

$$
\operatorname{Im}(\varepsilon)=\frac{C^{2}}{\omega^{2}\left(\mu_{\mathrm{r}}\right)}(k \alpha),
$$

where $\mu_{\mathrm{r}}$ is the relative permeability. For most crystalline materials $\mu_{\mathrm{r}}$ is very close to 1 at optical frequencies. On putting the value of $\operatorname{Im}(\varepsilon)$ in Eq. (11):

$$
\sigma=\frac{\alpha n c}{4 \pi}
$$

where $c$ is the velocity of light, $\alpha$ is the absorption coefficient and $n$ is the refractive index. The optical conductivity as a function of the frequency response of the material when irradiated with light was calculated by using the following relation and its value was found to be $1.4 \times 10^{7} \mathrm{~s}^{-1}$ at $1400 \mathrm{~nm}$. The electrical conductivity could also be estimated by optical method using the following relation [31]:

$$
\sigma_{\mathrm{e}}=\frac{2 \lambda \sigma}{\alpha} \text {. }
$$

The electrical conductivity was found to be $5.36 \times$ $10^{10} \Omega^{-1} \mathrm{~cm}^{-1}$ at $1400 \mathrm{~nm}$. The material is found to be transparent to all radiations in the wavelength range of 255-1400 nm. As there is no change in the transmittance in the entire visible region, it is an advantage as it 
is the key requirement for materials having NLO properties. For short wavelengths Rayleigh scattering of inhomogeneities becomes important and towards UV wavelengths electronic absorption starts to kick in. At around $1400 \mathrm{~nm}$ there is a strong absorption from $\mathrm{OH}$ groups. This leaves two windows for telecommunication with similarly low absorption: one around $1300 \mathrm{~nm}$ and another one around $1500 \mathrm{~nm}$. For larger wavelengths infrared absorption starts to increase.

\subsection{FTIR analysis}

The Fourier transform infrared spectrum of LCB crystal was obtained in the middle IR region between 4000 and $400 \mathrm{~cm}^{-1}$ using Bruker IFS $66 \mathrm{~V}$ FTIR spectrophotometer and the spectrum is shown in Fig. 4.

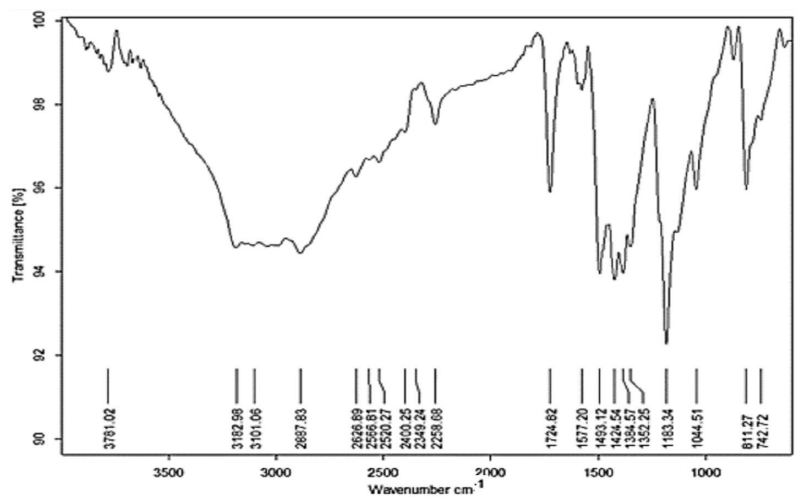

Fig. 4. FTIR spectrum of LCB.

The vibration spectrum of a molecule generally consists of two major regions: (i) group frequency region, (ii) finger print region. Group frequencies are vibrations that are associated with certain structural units such as $-\mathrm{CH}_{3}$, $-\mathrm{NH}_{2},-\mathrm{C} \equiv \mathrm{N}$ etc., and appear fairly at a constant region in the spectrum. The molecules having similar groups show their vibration in the form of bands called finger print region [32]. In the case of $\mathrm{LCB}, \mathrm{CO}_{2}$ symmetric and asymmetric stretching modes of L-cysteine are assigned to the bands at 1592 and $1384 \mathrm{~cm}^{-1}$, respectively. The bands at 1572,1424 , and $1384 \mathrm{~cm}^{-1}$ for Lcysteine are assigned to the $\mathrm{CO}_{2}^{-}$asymmetric and symmetric stretching modes. The presence of these bands showed that the adsorbed L-cysteine was to be in its zwitterionic state. The presence of $\mathrm{NH}_{3}^{+}$group is in fact supported by the assigned band at $1632 \mathrm{~cm}^{-1}$ for L-cysteine. The bands at $1184 \mathrm{~cm}^{-1}$ for L-cysteine as well supported the presence of the $\mathrm{NH}_{3}^{+}$group [33-36]. The peak at $3633 \mathrm{~cm}^{-1}$ is assigned to $\mathrm{OH}$ stretching vibration. For water molecules, the frequencies of the normal modes are 3730,1595 , and $3756 \mathrm{~cm}^{-1}$ according to Herzberg [37]. In the case of LCB, the symmetric and the asymmetric stretching of water give rise to absorption bands at 3633 , 1592 , and $3784 \mathrm{~cm}^{-1}$.

\subsection{Microhardness analysis}

Microhardness studies were carried out on the polished LCB crystal using MMT-X7B MATSUZAWA Vickers microhardness tester fitted with a diamond indenter.
The well polished LCB crystal with smooth and dominant face was placed on the platform of the Vickers microhardness tester. Now any one face of the crystal was indented gently by the loads varying from $1 \mathrm{~g}$ to $50 \mathrm{~g}$, for a dwell period of $3 \mathrm{~s}$ using Vickers diamond pyramid indenter attached to an incident ray research microscope. The Vickers indented impressions were approximately square in shape. The shape of the impression was structure dependent, face dependent and also material dependent. For a particular load, at least two welldefined impressions in the $X$-axis and the $Y$-axis were considered and the average of all the diagonals $d$ was considered. The Vickers hardness number $\left(H_{V}\right)$ was calculated using the standard relation

$$
H_{V}=1.8544 P / d^{2},
$$

where $P$ is the applied load in $\mathrm{kg}, d$ is the diagonal length of the indentation impression in micrometer and $H_{V}$ is in $\mathrm{kg} / \mathrm{mm}^{2}$. The variation of hardness number $H_{V}$ and load $P$ for LCB crystal is shown in Fig. 5a. The hardness increases gradually with the increase of load. Owing to the application of mechanical stress by the indenter, dislocations are generated locally at the region of the indentation. Higher hardness value of a crystal indicates that greater stress is required to form dislocation thus confirming greater crystalline perfection [38]. According to the Meyer law, the relation connecting the applied load is given by

$$
P=k_{1} d^{n}, \quad \log P=\log k+n \log d,
$$

where $n$ is the Meyer index or work hardening exponent and $k_{1}$ is the constant for a given material. The above relation indicates that $H_{V}$ should increase with load $P$ if $n<2$ and decrease with load $P$ when $n<2$. $n$ was determined from the slope of the plot that is shown in Fig. 5b. The value of $n$ for LCB was found to be 2.29. The low value of work hardening coefficient $n$ illustrates fewer defects in the grown crystal. The large value of $n$ indicates large effect of dislocations. According to Onitsch [39], $n$ should lie between 1 and 1.6 for harder materials and above 1.6 for softer materials. From the hardness study, the grown LCB crystal is found to be relatively soft material $[40,41]$. The material constant $k_{1}$ is calculated using the relation $k_{1}=P / d^{2}$ and it was found to be $6.18 \times 10^{5} \mathrm{~kg} / \mathrm{m}$. The elastic stiffness constant $\left(C_{11}\right)$ was calculated by using the Wooster empirical relation as $C_{11}=H^{7 / 4}$ [42]. The resistance pressure is defined as a minimum level of indentation load $(W)$ below which there is no plastic deformation [43]. Hays and Kendall proposed a relationship to calculate $W$ by the equation

$$
d^{n}=W / k_{1}+\left(k_{2} / k_{1}\right) d^{2} .
$$

The plot between $d^{n}$ and $d^{2}$ shown in Fig. 5c, gives a straight line having slope $\left(k_{2} / k_{1}\right)$ and intercepts $\left(W / k_{1}\right)$. From the graph, the slope and the intercept were found to be $3.03 \times 10^{2}$ and $6.38 \times 10^{5}$. From these values, the minimum level of indentation load $W$ was calculated to be $16.98 \mathrm{~g}$ and the value of $n$ was found to be 2.29. From this analysis, it is clear that below $16.98 \mathrm{~g}$, the crystal does not exhibit plastic deformation and from $16.98 \mathrm{~g}$ and 
upwards, it exhibits plastic deformation. Beyond $50 \mathrm{~g}$, cracks develop on the smooth surface of the crystal. The micro-indentation test is a useful method for studying the nature of plastic flow and its influence on the deformation of the material. Also, the hardness of the crystal is dependent on the type of chemical bonding, which may differ along the crystallographic direction [44]. Hardness is one of the important factors in selecting the processing (cutting, grinding, and polishing) steps of bulk in the fabrication of devices based on the crystals. It is therefore important to study the mechanical properties of NLO or organic crystals [45].

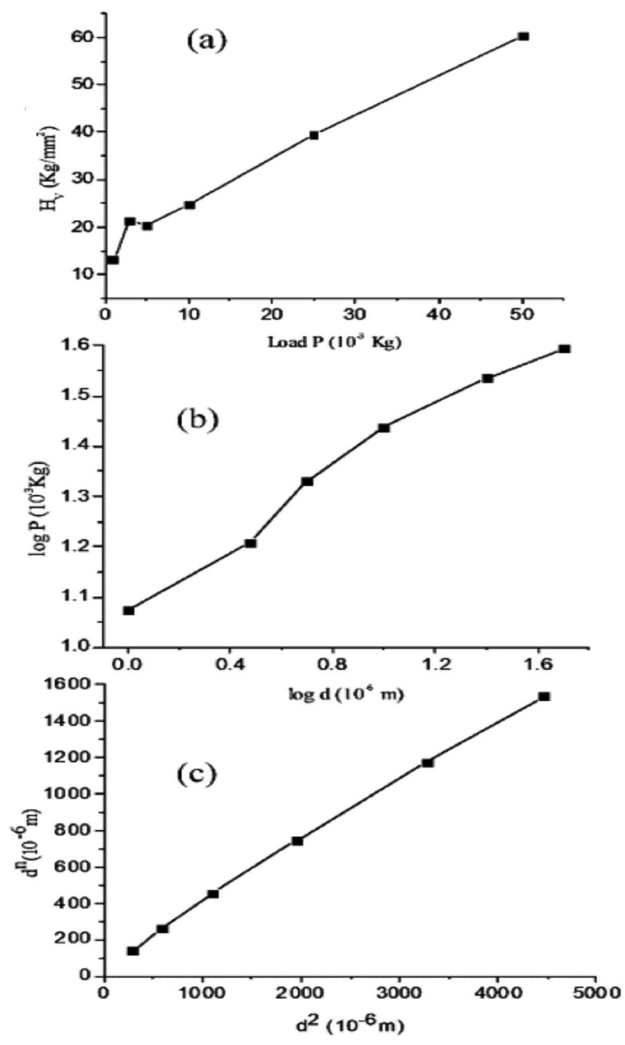

Fig. 5. (a) $H_{V}$ vs. $\operatorname{load} P$, (b) $\log P$ vs. $\log d$, (c) $d^{n}$ vs. $d^{2}$ of LCB.

\subsection{Thermal analysis}

The TGA and DTA are very important to find the thermal stability of the substance. Thermal analysis was carried out using SDT Q600V 8 build 95 simultaneous TGA/DTA analyzer in the nitrogen atmosphere at a heating rate of $20^{\circ} \mathrm{C} / \mathrm{min}$ in the temperature range 20 $1000^{\circ} \mathrm{C}$ for LCB crystal. A powder sample of $4.83 \mathrm{mg}$ was used for this investigation. From Fig. 6 there is observed a weight loss of about $20.01 \%$ in the temperature range $108.7-138.07^{\circ} \mathrm{C}$ which is mainly attributed to the decomposition of water molecules. It is evident that the compound LCB is stable up to the temperature $219.20^{\circ} \mathrm{C}$. The TGA curve also shows that there is a weight loss of about $17.42 \%$ in the temperature

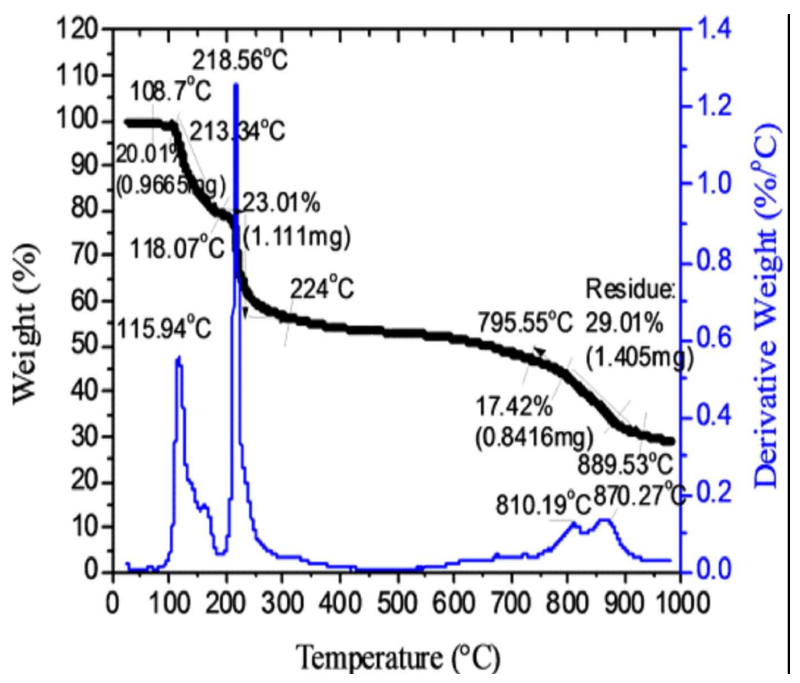

Fig. 6. TG-DTA curve of LCB.

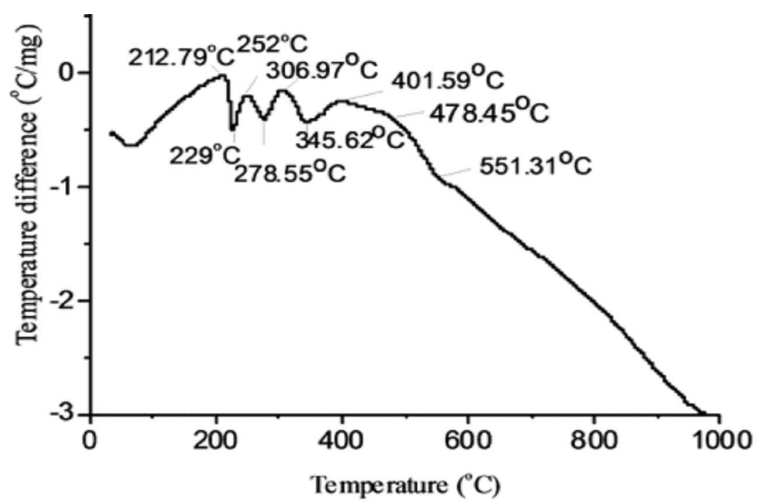

Fig. 7. DSC curve of LCB.

range $795.55-889.53{ }^{\circ} \mathrm{C}$ due to the liberation of volatile substance in the compound. The DTA curve shows an exothermic peak at $219.2^{\circ} \mathrm{C}$, which is the melting point of the substance. Then it undergoes an irreversible endothermic peak at $810.91{ }^{\circ} \mathrm{C}$ which shows the decomposition of the material [46]. The heat capacity at constant pressure, of LCB crystal was measured by DSC analysis in the temperature range $20-1000^{\circ} \mathrm{C}$ at the heating rate of $20^{\circ} \mathrm{C} / \mathrm{min}$ in the nitrogen atmosphere for the system calibration using SDT Q600V 8 build 95. Crystal weighing about $4.83 \mathrm{mg}$ was placed in a sealed alumina DSC pan. The DSC curve of LCB is shown in Fig. 7. There is no endothermic transition below $212{ }^{\circ} \mathrm{C}$; the material was proved to be stable in this region. It is the property observed for compounds, where the lattice force is more predominant than the covalent bonding forces in the molecules. Even the resistivity of the material against the thermal crack was visible evidence in this study. The DSC curve observed is smooth up to $212^{\circ} \mathrm{C}$.

\subsection{Dielectric studies}

Good quality single crystals of LCB having regular faces were selected for dielectric measurements. The crystal for the study was prepared from the larger ones by 
thinning them to the appropriate thickness. Suitably cut and polished LCB crystals (with known dimensions) were subjected to dielectric studies using HIOKI 353250 HITESTER LCR meter with a conventional four terminal sample holder. The crystal was prepared and mounted between the copper electrodes. In order to ensure good ohmic contact, the side of the crystal was coated with silver. The experiment was carried out for frequencies varying from $50 \mathrm{~Hz}$ to $5 \mathrm{MHz}$ and for different temperatures. The study of the dielectric constant $\left(\varepsilon_{\mathrm{r}}\right)$ and the dielectric loss $(\tan \delta)$ as a function of frequency and temperature helps us to know the various polarization mechanisms in the material. The dielectric constant $\left(\varepsilon_{\mathrm{r}}\right)$ could be calculated using the relation

$$
\varepsilon_{\mathrm{r}}=\frac{C t}{\varepsilon_{0} A},
$$

where $C$ is the capacitance, $t$ is the thickness and $A$ is the area of cross-section of the sample. It is observed that the dielectric constant decreases with increasing frequency and temperature as shown in Fig. 8. The dielectric constant is high at lower frequency region and decreases with increasing frequencies. The high value of dielectric constant at low frequencies is attributed to space charge polarization. At lower range of frequencies the dielectric constant decreases sharply with frequency becoming constant at larger frequencies [47, 48]. The polar-

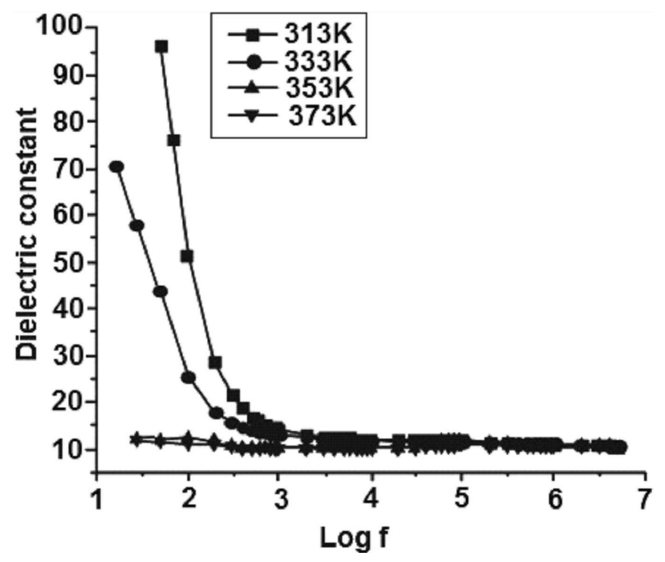

Fig. 8. $\log$ frequency vs. dielectric constant of LCB.

ization occurs due to the local displacement of electrons, which is the effect of the electronic exchange of the number of ions in the crystal. As the frequency increases, the electron exchange is unable to follow the electric field and the polarization becomes less dependent or independent of frequency. Variation of dielectric constant with temperature is generally attributed to the orientational polarization, crystal expansion, the presence of impurities and crystal defects. The thermal energy disrupts the ion dipole interaction which is responsible for the polarization at higher temperatures, causing the relaxation of polarization.

The dielectric loss is also studied as a function of frequency for different temperatures and is shown in Fig. 9.

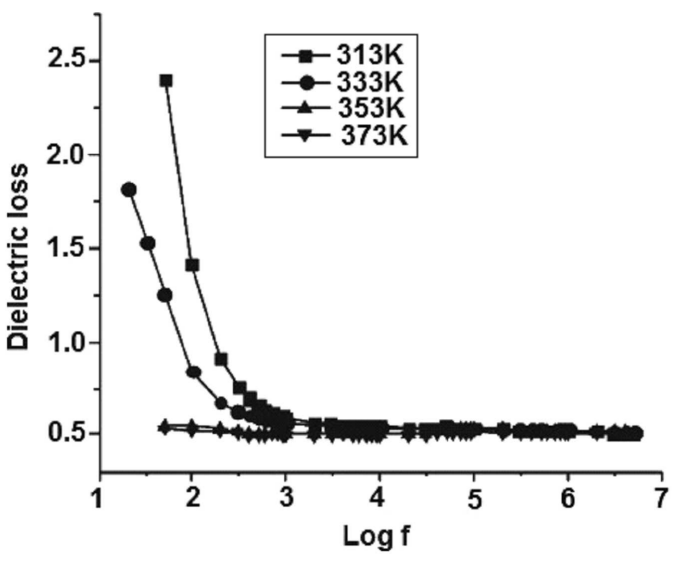

Fig. 9. $\log$ frequency vs. dielectric loss of LCB.

The low dielectric loss at high frequencies for the given crystal indicates very high purity of the material. This parameter is of vital importance for non-linear optical materials in their applications. These curves suggest that the dielectric loss is strongly dependent on the frequency of the applied field. The higher values of the dielectric loss at low frequencies originate from space charge polarization mechanism and the characteristic of low dielectric loss at high frequencies reveals that the grown crystal possesses relatively high optical quality with low defect density. The behaviour of low dielectric loss with high frequency for the crystal suggests that the crystal possesses enhanced optical quality with lesser defects and this parameter plays a vital role for the fabrication of non-linear optical devices $[49,50]$.

\subsection{Second harmonic generation}

The most widely used technique for confirming the SHG efficiency of NLO materials to identify the materials with non-centrosymmetric crystal structures is the Kurtz powder technique [51]. In this technique the powdered sample with an average particle size range 125$150 \mu \mathrm{m}$ is filled in a microcapillary tube about $1.5 \mathrm{~mm}$ diameter. Q-switched Nd:YAG laser emitting a fundamental wavelength of $1064 \mathrm{~nm}$ is used. The input laser energy incident on the sample is $2.5 \mathrm{~mJ} /$ pulse and a pulse width of $10 \mathrm{~ns}$ with a repetition rate of $10 \mathrm{~Hz}$ is made to fall normally on the sample. The standard NLO inorganic potassium dihydrogen phosphate (KDP) is used as the reference material. The emission of green light $(\lambda=532 \mathrm{~nm})$ from the LCB crystals confirms their noncentrosymmetric crystal structure. The second harmonic radiation generated by the randomly oriented microcrystals is focused by a lens and detected by a photomultiplier tube. The generation of the second harmonic is confirmed by a strong bright green emission emerging from the powdered sample. The relative conversion efficiency is calculated from the output power of the LCB crystals with reference to KDP crystal. It was observed from the results that the second harmonic signal was about $12.5 \mathrm{mV}$. But the standard KDP crystals gave an SHG of $10 \mathrm{mV} /$ pulse 
from the same input energy. Hence, it could be seen that the SHG efficiency of the LCB crystal was 1.25 times that of the pure KDP crystal. The generation of second harmonics was confirmed by the green colour of the output radiation.

\section{Conclusion}

Bulk sized L-cysteine hydrochloride monohydrate (LCB) single crystals were grown by slow evaporation technique. Single crystal XRD and powder XRD confirmed the lattice parameters. UV-visible studies and FTIR studies revealed the transmission range and the functional groups for the given material. The band gap value was found to be $4.80 \mathrm{eV}$. The optical constants such as the refractive index, the extinction coefficient and the electrical susceptibility were calculated to analyse the optical property. The Vickers hardness study confirmed that the material belonged to soft category. The TGA and DTA analysis showed that the crystal could be thermally stable up to $108.7^{\circ} \mathrm{C}$. The dielectric constant and the dielectric loss measurements were made at different temperatures and frequencies. The NLO study confirmed the SHG property of the material and it was found to be 1.25 times more than that of KDP. This material exhibited NLO behaviour remarkably due to its better optical and dielectric properties.

\section{References}

[1] J.L. Bredas, C. Adant, P. Tackx, A. Persoons, Chem. Rev. 94, 243 (1994).

[2] F. Zernike, J.E. Midwinter, Applied Nonlinear Optics, Wiley, New York 1973.

[3] N. Prasad David, J. Williams, Introduction to Nonlinear Optical Effect in Molecules and Polymers, Wiley, New York 1991.

[4] D.F. Eaton, Nonlin. Opt. Mater. Sci. 253, 281 (1991).

[5] P. Tanusri, K. Tanusree, B. Gabriele, Lara Rigi, Cryst. Growth Des. 3, 13 (2003).

[6] J.C. Brice, Crystal Growth Processes, Wiley, New York 1986.

[7] J.C. Brice, The Growth of Crystals from Liquids, Wiley, New York 1972.

[8] R.A. Laudise, The Growth of Single Crystals, Prentice Hall, Eaglewood Cliffs (NJ) 1970.

[9] W.D. Lawson, S. Neilson, Preparation of Single Crystals, Butterworths, London 1958.

[10] C. Chuangtian, W. Yicheng, L. Rukang, J. Cryst. Growth 99, 790 (1990).

[11] P. Becker, Adv. Mater. 10, 979 (1998).

[12] S. Stella Mary, S. Shahi Kirupavathy, P. Mythili, R. Gopalakrishnan, J. Spectrosc. 29, 1311 (2008).

[13] G. Pasupathi, P. Philominathan, Int. J. Electron. Eng. Res. 2, 79 (2010).

[14] C. Ramachandra Raja, G. Gokila, A. Antony Joseph, Spectrochim. Acta A 72, 753 (2009).
[15] S.A. Martin Britto Dhas, S. Natarajan, Optic Commun. 281, 457 (2008).

[16] M. Loganayaki, P. Murugakoothan, Asian J. Chem. 23, 5085 (2011).

[17] P.V. Prasad, T.K. Visweswara Rao, K. Ramanchandra Rao, C. Satya Kamal, T. Samuel, J. Spectrochim Acta A Mol Biomol Spectrosc. 136, 1950 (2014).

[18] K. Selvaraju, R. Valluvan, K. Kirubavathi, S. Kumararaman, Opt. Commun. 269, 230 (2007).

[19] G. Bhagavannarayana, P. Rajesh, P. Ramasamy, J. Appl. Cryst. 43, 1372 (2010).

[20] S. Suresh, Optik-Int. J. Light Electron Opt. 125, 950 (2014).

[21] S. Suresh, Optik-Int. J. Light Electron Opt. 125, 1223 (2014).

[22] S. Suresh, D. Arivuoli, J. Optoelectron. Biomed. Mater. 3, 63 (2011).

[23] P. Koteeswari, S. Suresh, J. Mater. 2014, 362678 (2014).

[24] P. Koteeswari, S. Suresh, P. Mani, J. Minerals Mater. Character. Eng. 11, 813 (2012).

[25] S. Suresh, Am. J. Opt. Photon. 2, 24 (2014).

[26] V. Gupta, A. Mansingh, J. Appl. Phys. 80, 1063 (1996).

[27] A. Lucarelli, S. Lupi, P. Calvani, P. Maselli, G. De Marzi, P. Roy, N.L. Saini, A. Bianconi, T. Ito, K. Oka, Phys. Rev. B 65, 054511 (2002).

[28] E.I. Ugwu, A.S. Olayinka, F.I. Olabode, J. Eng. Appl. Sci. 4, 126 (2009).

[29] U. Mizutani, Introduction to the Electron Theory of Metals, Cambridge University Press, Cambridge 2004, p. 320 .

[30] P. Sharma, S.C. Katya, J. Phys. D Appl. Phys. 40, 2115 (2007)

[31] S. Rajathi, K. Kirubavathi, K. Selvaraju, Arab. J. Chem. (2015), in press.

[32] V. Revathi, V. Rajendran, Int. J. Recent Sci. Res. 4, 1332 (2013).

[33] S.D. Ross, J. Mol. Spectrosc. 29, 131 (1969).

[34] G.D. Chryssikos, J. Raman Spectrosc. 22, 645 (1991).

[35] G.D. Chryssikos, J.A. Kapoutsis, A.P. Patsis, E.I. Kamitsos, Spectrochim. Acta A Mol. Spectrosc. 47, 1117 (1991).

[36] N.B. Clothup, Introduction to Infrared and Raman Spectroscopy, 2nd ed., Academic Press, London 1975.

[37] G. Herzberg, Infrared and Raman Spectra of Polyatomic Molecules, Van Nostrand, New York 1945.

[38] S. Suresh, R. Varatharajan, Int. J. Phys. Sci. 8, 1892 (2013).

[39] E.M. Onitsch, Microscope 95, 12 (1950).

[40] S. Suresh, J. Optoelectron. Adv. Mater. 6, 1174 (2012).

[41] S. Suresh, A. Ramanand, P. Mani, K. Murthyanand, J. Optoelectron. Biomed. Mater. 1, 129 (2010).

[42] W.A. Wooster, Rep. Prog. Phys. 16, 62 (1953).

[43] V. Gupta, K.K. Bamzai, P.N. Kotru, B.M. Wanklyn, Mater. Chem. Phys. 89, 64 (2005). 
[44] S. Anbukumar, S. Vasudevan, P. Ramasamy, Mater. Chem. Phys. 16, 125 (1987).

[45] G. Anandhababu, G. Bhagavannarayana, P. Ramasamy, J. Cryst. Growth 310, 1228 (2008).

[46] T. Uma Devia, N. Lawrence, R. Rameshbabu, S. Selvanayagam, H. Stoeckli-Evans, G. Bhagavannarayana, K. Ramamurthi, J. Miner. Mater. Character. Eng. 9, 495 (2010).

[47] S. Sagadevan, Optik-Int. J. Light Electron Opt. 125, 6746 (2014).
[48] S. Suresh, A. Ramanand, D. Jayaraman, P. Mani, J. Optoelectron Adv. Mater. 4, 1763 (2010).

[49] S. Sagadevan, P. Murugasen, J. Crystallizat Proc. Technol. 4, 992014.

[50] A. Firdous, I. Quasim, M.M. Ahmad, P.N. Kotru, Bull. Mater. Sci. 33, 377 (2010).

[51] S.K. Kurtz, T.T. Perry, J. Appl. Phys. 39, 3798 (1968). 